\title{
LA REVOLUCIÓN DE LA CIRUGÍA ROBÓTICA EN LATINO AMÉRICA Y LA FUTURA IMPLEMENTACIÓN EN EL SISTEMA DE SALUD DEL PERÚ
}

\author{
THE REVOLUTION OF ROBOTIC SURGERY IN LATIN AMERICA AND THE FUTURE IMPLEMENTATION \\ IN THE HEALTH SYSTEM OF PERU
}

Jorge A. Cornejo-Aguilar 1,2, José Cornejo 1,3, Mariela Vargas ${ }^{2,4}$, Raul Sebastian ${ }^{5}$

\begin{abstract}
Sr. Editor
La Cirugía ha marcado un hito en la historia, desde sus orígenes y durante muchos siglos, esta especialidad estaba en estrecha relación con la curación de heridos en combate, una gran evidencia se denota en el caso presentado durante los inicios de la Cirugía Abierta, con las intervenciones llamadas Trepanaciones Craneanas que fueron realizadas en los años 700 a.C. - 200 d.C. por la Cultura Paracas en el Perú; con el transcurso del tiempo se fueron perfeccionando diversas técnicas que se complementaron con el uso de nuevos instrumentos y el desarrollo de la tecnología dando lugar a una revolución en las diversas especialidades quirúrgicas ${ }^{1}$.

Miles de años después, se le atribuye al cirujano alemán George Kelling la primera exploración abdominal con Cirugía Laparoscópica gracias a la creación del endoscopio en el año 1901; posteriormente, en Latino América se realizó la primera colecistectomía laparoscópica en 1990 por el cirujano Leopoldo Gutiérrez en México, y en Perú fue efectuada en Octubre de 1990 por el Dr. Mario del Castillo Irygoyen en el Hospital Nacional Cayetano Heredia, cabe mencionar también al Dr. Gustavo Salinas Sedó quien es pionero e influyente en esta técnica en el Perú y además es miembro fundador de la Sociedad Peruana de Cirugía Laparoscópica y de la Sociedad Latinoamericana de Cirugía Endoscópica, actualmente es un procedimiento que brinda al cirujano la oportunidad de realizar las mismas operaciones que la Cirugía Abierta, pero con incisiones más pequeñas y mejores resultados estéticos².

No obstante, el acontecimiento que ha marcado la Revolución de la Industria Biomédica ha sido la introducción de la Cirugía Robótica; procedimiento en donde concuerdan la tecnología avanzada, la inteligencia artificial y los procesos de manufactura, con el fin de diseñar equipamiento médico e instrumental más sofisticados para realizar intervenciones quirúrgicas de mayor precisión, como es el caso del dispositivo Vessel Sealer que permite hemostasia perfecta con daño térmico mínimo y los instrumentos Endowrist que mejoran la destreza de la mano-muñeca humana para efectuar la simulación de 7 grados de libertad y son de dimensiones muy pequeñas, además mencionar a un equipo que cuenta con capacidad de fluorescencia que detecta la vascularidad de una anastomosis llamado Firefly ${ }^{3}$. Figura 1.

A nivel mundial, el sistema de cirugía robótica más utilizado es el da Vinci® con sus plataformas $\mathrm{Si}$ y $\mathrm{Xi}_{\text {, }}$ el uso de estos presenta ventajas en comparación a la cirugía laparoscópica tales como menor pérdida y requerimiento de transfusión de sangre, menor dolor postoperatorio, estancias hospitalarias y tiempos de recuperación más cortos; a expensas de más largos tiempos operatorios y un costo mayor comparado con la técnica laparoscópica aunque con nueva tecnología y más demanda en su uso los tiempos se acortarían
\end{abstract}

\footnotetext{
Surgical Engineering Society

2 Facultad de Medicina Humana. Universidad Ricardo Palma, Lima-Perú.

${ }^{3}$ IEEE Engineering in Medicine and Biology Society.

${ }^{4}$ Instituto de Investigación en Ciencias Biomédicas. Universidad Ricardo Palma. Lima, Perú.

5 Medical Staff at George Washington University Hospital, EE.UU.
}

Citar como: Jorge A. Cornejo-Aguilar, José Cornejo, Mariela Vargas, Raul Sebastian. La revolución de la cirugía robótica en latino américa y la futura implementación en el sistema de salud del Perú. [Cartas al Editor].2019;19(1):108-111. (Enero 2018). DOI 10.25176/RFMH.v19.n1.1800 
y los costos también bajarían; asimismo, los cirujanos pueden beneficiarse con una ergonomía mejorada y disminución de esfuerzo, pudiendo ser operado de manera semi-autónoma desde una mesa cercana o desde distancias lejanas otorgando la posibilidad de realizar Telecirugía, dado que se tiene el control total de los brazos robóticos a través de una consola con visión 3D de profundidad anatómica para que las maniobras del cirujano sean más firmes, precisas y seguras eliminando el temblor de la mano humana ${ }^{4}$. Figura 2.

Esta técnica se adoptó rápidamente en hospitales de los Estados Unidos teniendo como pionero en esta técnica a Dr. David Samadi, y el Dr. Pier Cristoforo Giulianotti en Europa como tratamiento para una gran variedad de enfermedades y patologías, llegando así a Latino América en el año 2005 teniendo como sede al Hospital de Clínicas en Argentina, donde se realizó una cirugía en un paciente que padecía acalasia, procedimiento que fue transmitido en directo durante el $76^{\circ}$ Congreso Argentino de Cirugía 5 .

En Septiembre de 2018, Sao Paulo - Brasil, durante el XV Congreso Paulista de Urología fue presentada la cantidad de Sistemas Quirúrgico Robóticos da Vinci ${ }^{\circledR}$ que se encuentran operativos en Latino América, siendo el Perú uno de los países que carecen de esta tecnología5. Tabla 1.

Tabla 1. Cantidad de Sistemas Quirúrgicos Robóticos da Vinci $^{\circledR}$ en Latino.

\begin{tabular}{lc}
\multicolumn{1}{c}{ LATINO AMÉRICA } & $\begin{array}{c}\text { SISTEMA QUIRÚRGICO } \\
\text { ROBÓtICO DA VINCI }{ }^{\circ *}\end{array}$ \\
\hline Crasil & 37 \\
México & 10 \\
Chile & 7 \\
Argentina & 6 \\
Venezuela & 5 \\
Colombia & 5 \\
Panamá & 1 \\
Uruguay & 1 \\
República Dominicana & 1 \\
Perú & 0 \\
Otros & 15 \\
Total & 88 \\
\hline
\end{tabular}

* El Sistema Robótico Quirúrgico da Vinci $^{\circledR}$ es Fabricado por la Empresa Intuitive Surgical, Inc ${ }^{\otimes}$
La técnica quirúrgica llevada a cabo con el sistema da Vinci $^{\circledR}$ se caracteriza por ser mínimamente invasiva, encontrándose diversas aplicaciones clínicas tales como la prostatectomía radical y nefrectomía en el área urológica; en Ginecología permite la realización de histerectomías, en cirugía Cardiotorácica permite la reparación de válvula mitral y lobectomía, en cirugía Gastrointestinal la realización de resección pancreática y colecistectomía y en Pediatría el manejo quirúrgico de enfermedades congénitas cardiacas ${ }^{6}$.

La implementación del Sistema Quirúrgico Robótico da Vinci ${ }^{\circledast}$ en el Perú, podría otorgar una mejor calidad de atención, cuidado, diagnóstico y tratamiento de los pacientes; además de la posible integración de un nuevo miembro al equipo de salud quirúrgico -el Ingeniero Biomédico- con la finalidad de brindar una eficiente gestión de tecnologías en salud durante los procedimientos.

El introducir dicha tecnología en el Perú, implica un análisis de costo- accesibilidad; en Reino Unido, tomando como caso guía la cirugía de extirpación de próstata, mediante la cirugía robótica tiene un costo total que oscila entre US $\$ 2000-\$ 39,215$, realizado mediante laparoscópica se encuentra en el rango de US $\$ 740-\$ 29,771$ y la prostatectomía radical abierta entre US $\$ 1870-\$ 31,518$; esta diferencia se debe al costo de compra del robot, instrumentos y mantenimientos (7), el cual es un precio elevado para el sueldo mínimo de un trabajador peruano. A pesar de ello, su implementación podría establecer mejoras significativas en la salud de la población.

El costo de los procedimientos desarrollados en un inicio será notablemente elevado, sin embargo, con el transcurso de su uso y al enmarcar todos sus beneficios, sumándose a la inversión de instituciones nacionales e internacionales y a la cobertura por parte de las Aseguradoras de Salud, se podrá tener mayor accesibilidad a este sistema robótico; por lo tanto, la implementación del Robot da Vinci ${ }^{\circledR}$, tendrá un efecto de costo - beneficio a futuro.

El impacto de esta tecnología en el Sistema Salud del Perú será notable, lo cual se ve reflejado en un mejor desarrollo de los procedimientos quirúrgicos debido a la precisión que se le brinda al operador a través de un mejor alcance y rango de movimiento; a su vez, cabe resaltar que países con una situación económica similar a la nuestra tiene acceso a este sistema quirúrgico robótico, por lo que es fundamental su implementación para que de esta manera se pueda revolucionar la medicina peruana. 


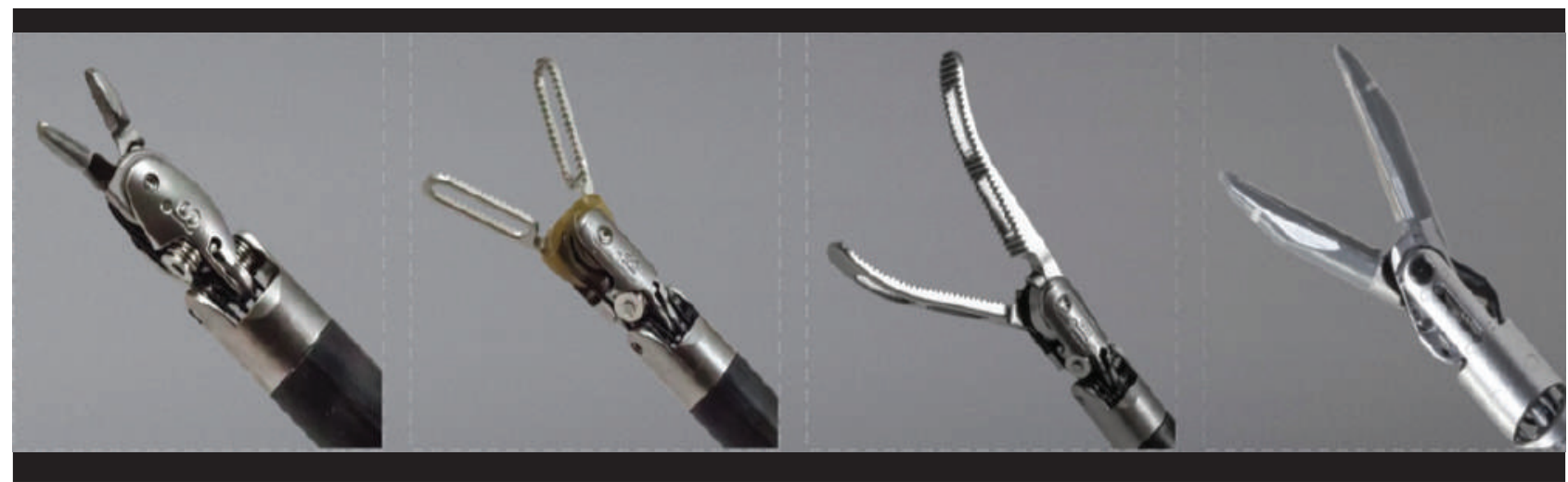

Figura 1. Instrumentos Endowrist Needle Driver, Forceps, Grasper, Vessel Sealer (izquierda a derecha). Intuitive Surgical, Inc ${ }^{\circledR}$. Imagen disponible en: https://www.intuitivesurgical.com/images/on-site-banners/1008471rB-EU_Xi_ IA_Catalog.pdf

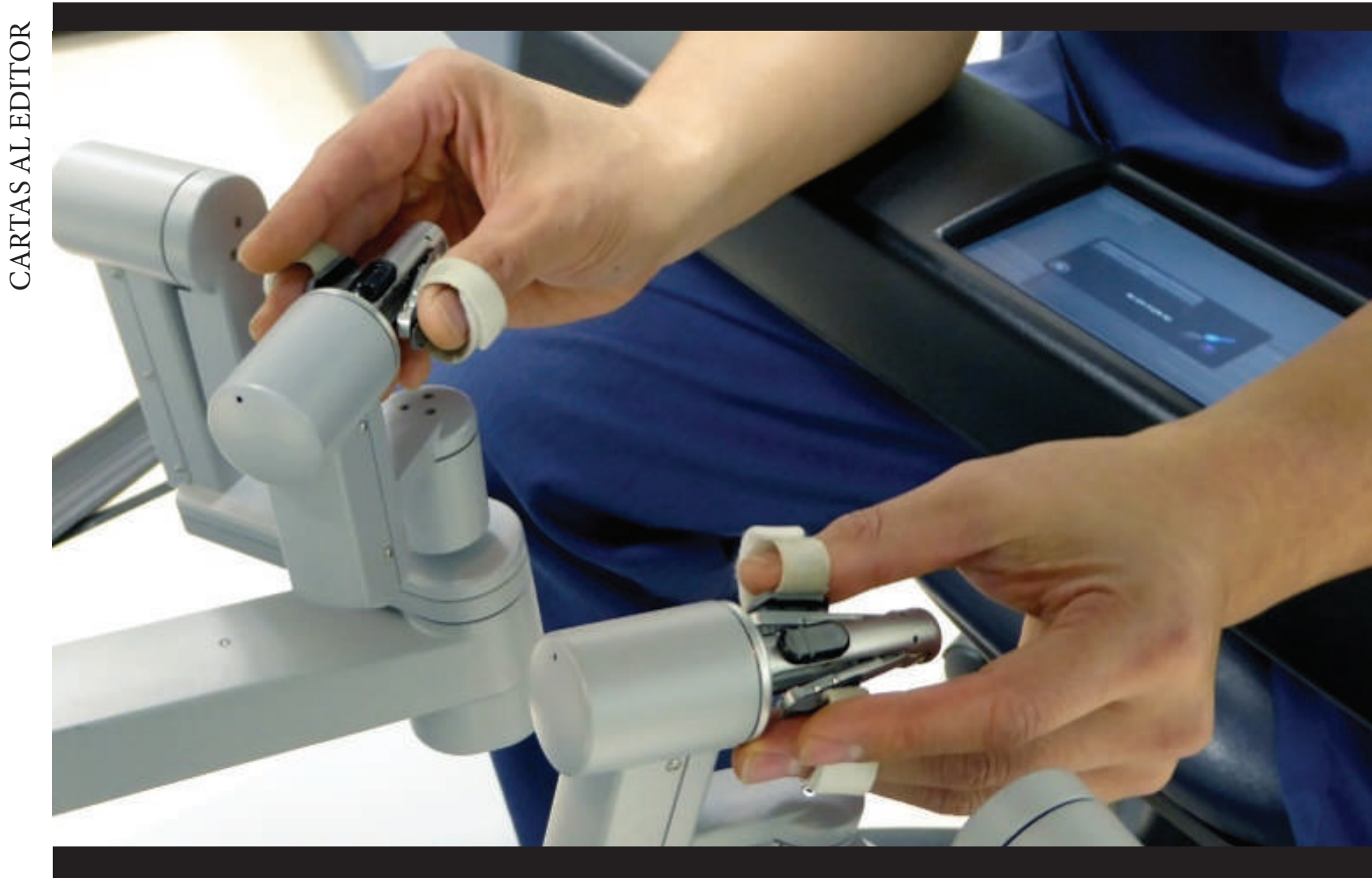

Figura 2. Control de Brazos Robóticos con la Consola del Cirujano del da Vinci. Intuitive Surgical, Inc ${ }^{\circledR}$. Imagen disponible en:https://sius.davincisurgerycommunity.com/detail/videos/p9_rc_surgeon-console/video/5622753693001/ instrument-controls:-using-the-masters?autoStart=true

Contribuciones de autoría: Los autores participaron en la idea, diseño, redacción, revisión crítica y aprobación de la versión final del artículo.

Financiamiento: Autofinanciado.

Conflicto de interés: Los autores declaran no tener conflicto de interés en la publicación de este artículo.

Recibido: 13 de noviembre del 2018

Aprobado: 21 de diciembre del 2018

\section{Correspondencia: Jorge A. Cornejo Aguilar} Dirección: Calle Galicia 369. Lima, Perú Celular: +51 993519893

Correo:jcornejo_17@ieee.org 


\section{REFERENCIAS BIBLIOGRÁFICAS}

1. Kushner DS, Verano JW, Titelbaum AR. Trepanation Procedures/Outcomes: Comparison of Prehistoric Peru with Other Ancient, Medieval, and American Civil War Cranial Surgery. World Neurosurgery. 2018; 114: 245-51. Disponible en: https://www.ncbi.nlm.nih.gov/pubmed/29604358

2. García Ruiz A, Gutiérrez Rodríguez L, Cueto García J. Evolución histórica de la cirugía laparoscópica. Cirugía Endoscópica. 2016; 17(2): 93-106. Disponible en: http://www.medigraphic.com/pdfs/endosco/ce-2016/ce162i.pdf

3. Feußner $H$, Ostler D, Wilhelm D. Robotics and augmented reality: Current state of development and future perspectives. Chirurg. 2018; 89(10): 760-8. Disponible en: https://www.ncbi.nlm.nih.gov/pubmed/30132168

4. Ho C, Tsakonas E, Tran K, Cimon K, Severn M, Mierzwinski-Urban M, et al. Robot-Assisted Surgery Compared with Open Surgery and Laparoscopic Surgery: Clinical Effectiveness and Economic Analyses. Ottawa: Canadian Agency for Drugs and Technologies in Health. 2011. (CADTH Technology Report, No. 137.). Disponible en: https://www.ncbi.n/m.nih.gov/books/ NBK168912/
5. Perez MC, Cesar Santos A, Betoni Guglielmetti G, Guillonneau B, Ibrahim Mitre A.

Debate: Prostatectomia Radical Laparoscópica na era da Robótica. Ponencia presentada en el XV Congreso Paulista de Urología, São Paulo Brasil. 2018. Disponible en:http://www.congressopaulistadeuro2018.com. br/programacao/index4_porsala_track_timeline.php?dt_sel=2018-0906\#2018-09-06

6. Bishoy Morris. Robotic surgery: applications, limitations, and impact on surgical education. MedGenMed: Medscape General Medicine. 2005 7(3): 72. Disponible en: https://www.ncbi.nlm.nih.gov/pmc/articles/ PMC1681689/

7. Ahmed K, Ibrahim A, Wang TT, Khan N, Challacombe B, Khan MS, Dasgupta P. Assessing the cost effectiveness of robotics in urological surgery - a systematic review. BJU International. 2012. 110(10): 1544-56. Disponible en: https://www.ncbi.nlm.nih.gov/pubmed/22443296

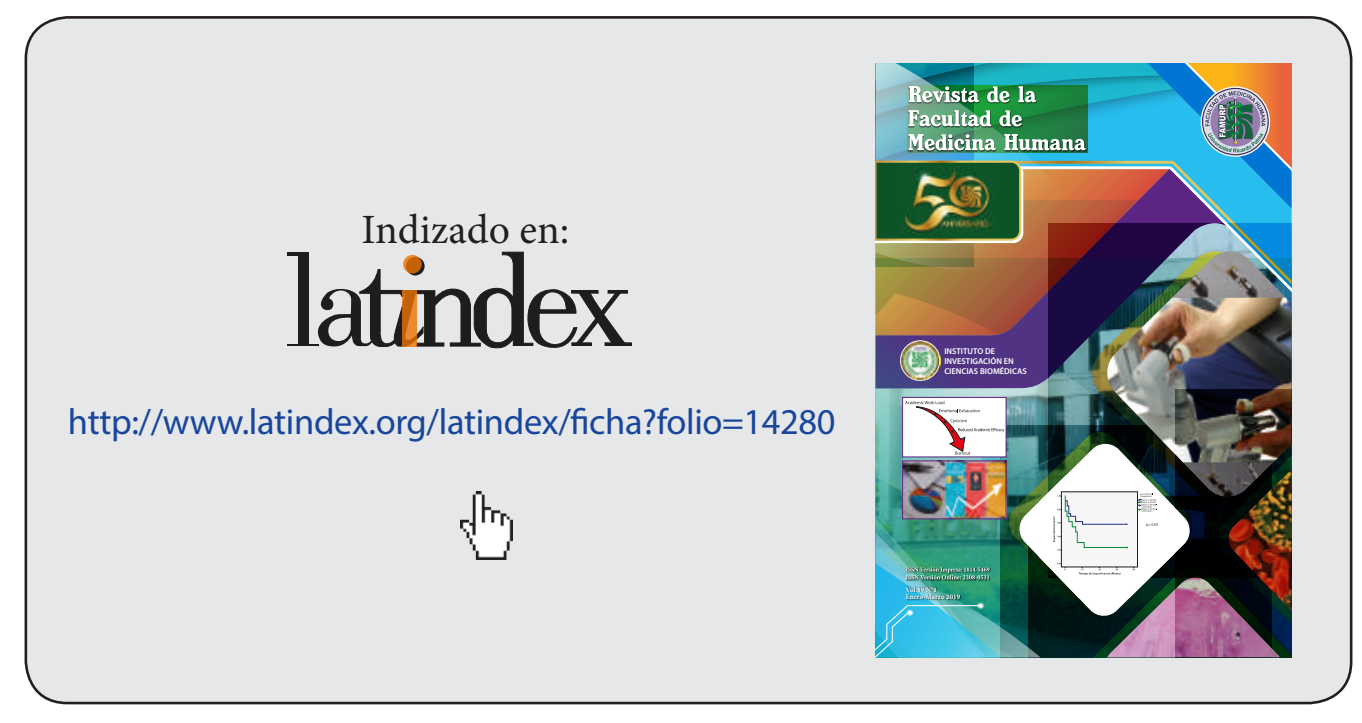

